doi: $10.13108 / 2021-13-2-107$

\title{
DIFFERENTIAL SUBSTITUTIONS FOR NON-ABELIAN EQUATIONS OF KDV TYPE
}

\author{
V.E. ADLER
}

\begin{abstract}
The work is devoted to constructing differential substitutions connecting the non-Abelian KdV equation with other third-order evolution equations. One of the main results is the construction of a non-Abelian analog of the exponential Calogero-Degasperis equation in a rational form. Some generalizations of the Schwarzian KdV equation are also obtained. Equations and differential substitutions under study contain arbitrary nonAbelian parameters. The construction method is based on the auxiliary linear problem for $\mathrm{KdV}$, in which the usual spectral parameter is replaced by a non-Abelian one. The wave function, corresponding to a fixed value of this parameter, also satisfies a certain evolution equation. Passing to the left and right logarithmic derivatives of the wave function leads one to two versions of the modified $\mathrm{KdV}$ equation. In addition, a gauge transformation of the original linear problem leads to a linear problem for one of these versions, mKdV-2. After that, the described procedure is repeated, and the resulting evolution equation for the wave function contains already two arbitrary non-Abelian parameters. For the logarithmic derivative, we obtain an analog of the Calogero-Degasperis equation, which is thus a second modification of the KdV equation. Combining the found Miura-type transformations with discrete symmetries makes it possible to obtain chains of Bäcklund transformations for the modified equations.
\end{abstract}

Keywords: non-Abelian equation, Lax pair, Miura transformation.

Mathematics Subject Classification: 35Q53, 37K30, 37K35

\section{INTRODUCTION}

Equations with non-commutative (for example, matrix) unknowns are an important object of study in the theory of integrable systems. One of the first examples was the non-Abelian Korteweg-de Vries equation (KdV)

$$
u_{t}=u_{x x x}-3 u u_{x}-3 u_{x} u
$$

and its modification $(\mathrm{mKdV})$

$$
f_{t}=f_{x x x}-3 f^{2} f_{x}-3 f_{x} f^{2}
$$

The inverse scattering method, families of exact solutions, Hamiltonian structures and Darboux-Bäcklund transformations for these equations were studied in [1], [2], [3], 4], 5], [6] and other works. In [7], another version of mKdV was introduced

$$
v_{t}=v_{x x x}+3\left[v, v_{x x}\right]-6 v v_{x} v,
$$

which is related with (1.1) by an implicit change [8]. Currently, non-Abelian analogs are found for a large number of various integrable models, including evolution and hyperbolic systems, 3D equations, (semi)-discrete and ordinary differential equations. A deep, but still far from being complete, presentation of the subject is contained in the book [9]. A fairly wide family of nonlinear Schrödinger and Boussinesq type systems being polynomial and linear with respect to

V.E. Adler, Differential substitutions for non-Abelian equations of KdV type.

(C) V.E. ADLER. 2021.

The reported study was funded by RFBR and SC RA, project number 20-52-05015.

Submitted March 10, 2021. 
the derivatives was studied in [10], and in most cases there were two non-Abelian counterparts corresponding to one scalar system, like in the case of (1.1) and (1.2).

At the same time, there are many blank spots in the non-commutative theory, for example, no classification is known for integrable equations of the KdV type

$$
u_{t}=u_{x x x}+F\left(x, u, u_{x}, u_{x x}\right) .
$$

In the scalar setting, such classification was obtained long ago in the framework of the symmetry approach [11], [12, but in the non-Abelian case we even do not know exactly which equations from the scalar list admit a generalization. In addition to the above equations, one can find in the literature non-Abelian analogs of the potential mKdV equation (pmKdV) and the Schwarzian KdV equation (SKdV), see, for instance, [9, Ch. 3.9] and [13], and the author does not know any other studies. Moreover, only homogeneous equations without parameters were studied. Meanwhile, recently it was observed [14] that equation (1.2) can be generalized by adding lower-order terms with an arbitrary non-Abelian constant, which is important while constructing a self-similar reduction and leads one to a new version of the non-Abelian Painlevé-II equation.

The purpose of this work is to enlarge the list of examples of type $(1.3)$ by use of differential substitutions. A similar problem was posed in [15], where, however, no new equations were obtained.

Recall that from the point of view of substitutions, all scalar integrable equations (1.3) are divided into three subclasses [16]. One class includes the linearizable equations, the second one contains equations related to $\mathrm{KdV}$, and the third class consists of one isolated KricheverNovikov equation [17]

$$
z_{t}=z_{x x x}-\frac{3\left(z_{x x}^{2}-R(z)\right)}{2 z_{x}}+\alpha z_{x}, \quad R^{(5)}(z)=0,
$$

where $R$ is a polynomial of degree 3 or 4 with simple roots; the case of multiple roots reduces to $\mathrm{KdV}$. For equations related to KdV, the basic sequence of Miura type transformations is as follows:

$$
\begin{aligned}
u_{t}= & u_{x x x}-6 u u_{x}, \\
& \uparrow \quad u=f_{x}+f^{2}+\beta \\
f_{t}= & f_{x x x}-6\left(f^{2}+\beta\right) f_{x} \\
& \uparrow \quad 2 f=\frac{p_{x}+p^{2}+\alpha}{p} \\
p_{t}= & p_{x x x}-3 \frac{p_{x} p_{x x}}{p}+\frac{3 p_{x}^{3}}{2 p^{2}}-\frac{3}{2}\left(p+\frac{\alpha}{p}\right)^{2} p_{x}-6 \beta p_{x}, \\
& \uparrow \quad 2 p=\frac{\sqrt{w_{x}^{2}+4 R(w)}-w_{x}}{w-\gamma}, \\
w_{t}= & w_{x x x}-\frac{3 w_{x}\left(w_{x x}+2 R^{\prime}(w)\right)^{2}}{2\left(w_{x}^{2}+4 R(w)\right)}+6(2 w-\beta) w_{x},
\end{aligned}
$$

where $R(w)=\left(w^{2}-\gamma^{2}\right)(w+\gamma+\alpha)$. This "tower of modifications" and several simpler substitutions like the introduction of a potential cover almost all equations related to $\mathrm{KdV}$; the only exception is equation (1.4) with one double root and it is related to KdV by a third-order substitution which can not be decomposed into simpler ones. Note that each modification adds an arbitrary parameter, which is important for obtaining equations of general form. In the non-Abelian setting, we can expect that some parameters may be non-scalar. 
One of results of this paper is the non-Abelian analog of equation (1.7) which is the rational form of the Calogero-Degasperis equation [18], [19]. It is likely that equation (1.8), also introduced in [18], and equation (1.4) have no non-Abelian counterparts for generic polynomials $R$. However, there exist analogs for two degenerate cases of $(1.4)$ corresponding to $R=z^{2}$ and $R=1$.

The list of non-Abelian equations and substitutions presented in the next section does not pretend to be complete; it may well be that analogs of some other scalar equations can be added to this scheme. All substitutions can be verified by straightforward calculations. A more demonstrative proof based on the derivation of substitutions from auxiliary linear problems is given in Section 3.

It should be noted that the above tower of modifications for scalar equations can also be constructed based on the Bäcklund transformations, as shown by Yamilov [20], [21], see also [22], [23]. Unfortunately, this method does not work in the non-Abelian setting. However, the differential substitutions still can be used to generate the Bäcklund transformations; this is briefly discussed in Section 4.

\section{GRAPH OF SUBSTITUTIONS}

We will construct differential substitutions between the following equations:

$$
\begin{array}{rlrl}
u_{t} & =u_{x x x}-3 u u_{x}-3 u_{x} u, & \mathrm{KdV} \\
w_{t} & =w_{x x x}-3 w_{x}^{2}, & \mathrm{pKdV} \\
f_{t} & =f_{x x x}-3 f^{2} f_{x}-3 f_{x} f^{2}-6 \alpha f_{x}, & \operatorname{mKdV}_{1}(\alpha) \\
v_{t} & =v_{x x x}+3\left[v, v_{x x}\right]-6 v v_{x} v-3\left(v_{x}+v^{2}\right) a-3 a\left(v_{x}-v^{2}\right), & \operatorname{mKdV}(a) \\
y_{t} & =y_{x x x}-3 y_{x x} y^{-1} y_{x}-3 y_{x} a-3 y a y^{-1} y_{x}, & \operatorname{pmKdV}(a) \\
p_{t}=(D-\operatorname{ad} p)\left(p_{x x}-\frac{3}{2}\left(p_{x}+p^{2}-a\right) p^{-1}\left(p_{x}-p^{2}+a\right)+\left[p, p_{x}\right]-2 p^{3}-6 \beta p\right), & \mathrm{CD}(a, \beta) \\
q_{t}=q_{x x x}-\frac{3}{2} D\left(\left(q_{x}-c\right) q^{-1}\left(q_{x}+c\right)\right), & \mathrm{CD}(c) \\
z_{t}=z_{x x x}-\frac{3}{2}\left(z_{x x}-a z+z b-c\right) z_{x}^{-1}\left(z_{x x}+a z-z b+c\right)-3 a z_{x}-3 z_{x} b . & \operatorname{SKdV}(a, b, c)
\end{array}
$$

Here $D$ denotes the derivative with respect to $x$, field variables $u, \ldots, z$ and constants $a, b, c$ belong to a free associative algebra $\mathcal{A}$ over $\mathbb{C}$ with unit 1 , and $u^{-1}$ denote the inverse element for $u$. For the sake of clarity, one can assume that $\mathcal{A}$ is the algebra of matrices of some arbitrary fixed size. Equations $\mathrm{mKdV}_{1}(\alpha)$ and $\operatorname{CD}(a, \beta)$ also contain scalar constants $\alpha, \beta \in \mathbb{C}$. These parameters can be set to 0 by the Galilean transform, but we do not do this since they are involved in the substitutions and related Bäcklund transformations. In what follows, we identify $\alpha$ with $\alpha 1 \in \mathcal{A}$, which gives meaning for expressions like $u+\alpha$.

For each equation, the order of factors in monomials can be reversed by passing to the new operation of multiplication $a \circ b=b a$ on $\mathcal{A}$. The resulting equation will be called transposed by analogy with the matrix case. It is natural to regard it as equivalent to the original equation. The above equations have the property of invariance with respect to the transposition, possibly up to some additional involutions like the sign change (in $\operatorname{pmKdV}$, we apply $y \rightarrow y^{-1}$ ) or changing the parameters.

Remark 2.1. In the scalar case, equation $C D(\alpha, \beta)$ coincides with (1.7), the rational form of Calogero-Degasperis equation. Passing to the exponents brings it and $C D_{0}(\gamma)$ to the original 

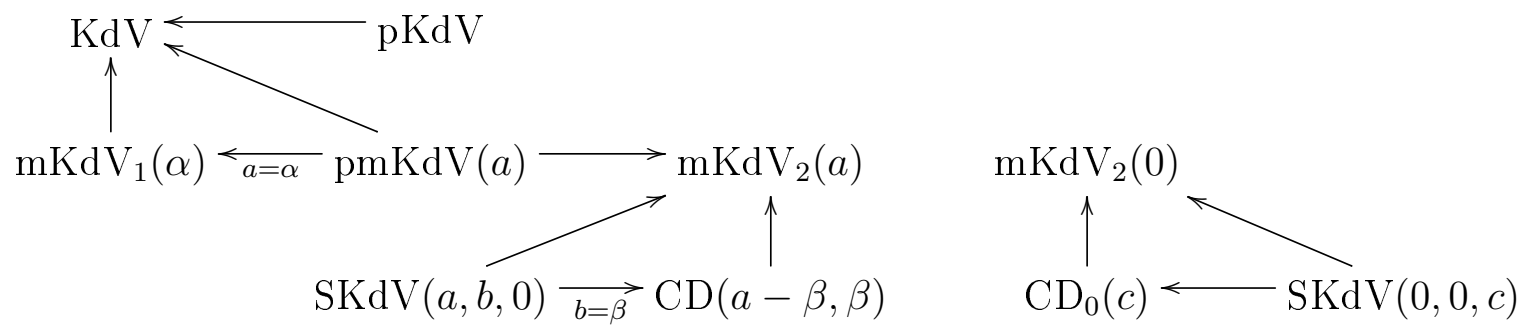

FiguRE 1. Substitutions chart

form from [18], [19]:

$$
\begin{gathered}
P_{t}=P_{x x x}-\frac{1}{2} P_{x}^{3}-\frac{3}{2}\left(e^{P}+\alpha e^{-P}\right)^{2} P_{x}-6 \beta P_{x}, \quad p=e^{P}, \\
Q_{t}=Q_{x x x}-\frac{1}{2} Q_{x}^{3}-\frac{3 \gamma^{2}}{2} e^{-2 Q} Q_{x}, \quad q=e^{Q} .
\end{gathered}
$$

The same change transforms the scalar equation $p m K d V(\alpha)$ to the usual polynomial form

$$
Y_{t}=Y_{x x x}-2 Y_{x}^{3}-6 \alpha Y_{x}, \quad y=e^{Y} .
$$

Remark 2.2. Comparing the above equations for $Q$ and $Y$, we see that $C D_{0}(0)$ is actually another non-Abelian analog of pmKdV. This equation was studied in [13]. One more interesting analog of pmKdV on Jordan algebras was obtained in [24, Eq. (2.4)]. In contrast to $C D_{0}(0)$, it involves the operator $M_{q}^{-1}$ instead of $q^{-1}$, where $M_{q}$ is the multiplication operator in the Jordan algebra. Since any associative algebra $\mathcal{A}$ turns into a Jordan algebra with respect to the operation $a \circ b=\frac{1}{2}(a b+b a)$, such equation can be defined on $\mathcal{A}$ as well, if we allow expressions involving $\left(L_{q}+R_{q}\right)^{-1}$, where $L_{q}$ and $R_{q}$ are operators of the left and right multiplication in $\mathcal{A}$.

Remark 2.3. Scalar equation $S K d V(a, b, c)$ is actually the degenerate case of KricheverNovikov equation (1.4) with $R=((a-b) z+c)^{2}$. The case $R=0$ is the Schwarzian $K d V$ equation, and we keep this name for the entire family of equations.

The following proposition is the main result of the paper.

Proposition 2.1. The above non-Abelian equations are related by substitutions

$$
\begin{aligned}
\operatorname{pKdV} & \rightarrow \mathrm{KdV}: & & =w_{x}, \\
\operatorname{pmKdV}(\alpha) & \rightarrow \operatorname{mKdV}_{1}(\alpha): & & =y_{x} y^{-1}, \\
\operatorname{mKdV}_{1}(\alpha) & \rightarrow \mathrm{KdV}: & u & =f_{x}+f^{2}+\alpha, \\
\operatorname{pmKdV}(a) & \rightarrow \mathrm{KdV}: & u & =y_{x x} y^{-1}+y a y^{-1}, \\
\operatorname{pmKdV}(a) & \rightarrow \operatorname{mKdV}_{2}(a): & v & =y^{-1} y_{x}, \\
\operatorname{SKdV}(a, \beta, 0) & \rightarrow \mathrm{CD}(a-\beta, \beta): & p & =z_{x} z^{-1}, \\
\mathrm{CD}(a-\beta, \beta) & \rightarrow \operatorname{mKdV}_{2}(a): & v & =-\frac{1}{2}\left(p_{x}+p^{2}-a+\beta\right) p^{-1}, \\
\operatorname{SKdV}(0,0, c) & \rightarrow \mathrm{CD}_{0}(c): & & =z_{x}, \\
\mathrm{CD}_{0}(c) & \rightarrow \operatorname{mKdV}_{2}(0): & v & =-\frac{1}{2}\left(q_{x}-c\right) q^{-1}, \\
\operatorname{SKdV}(a, b, c) & \rightarrow \operatorname{mKdV}_{2}(a): & v & =-\frac{1}{2}\left(z_{x x}-a z+z b-c\right) z_{x}^{-1} .
\end{aligned}
$$

Schematically, these substitutions are shown in Figure 1. We note that the substitution 2.2 from $\operatorname{pmKdV}(a)$ is valid only for the scalar value of parameter $a=\alpha$, so the substitution (2.4) is more general than the composition of $(2.2)$ and (2.3). Similarly, the substitutions (2.6) and (2.8) from $\operatorname{SKdV}(a, b, c)$ do not work for arbitrary $(a, b, c)$, so the substitution 2.10 is more general than the compositions of (2.6) and (2.7) or of $(2.8)$ and $(2.9)$. 
It is worth noting that if we consider the complete graph of substitutions for scalar equations (the sequence $(1.8) \rightarrow \cdots \rightarrow(1.5)$ from Introduction is a part of this graph) then the $\mathrm{KdV}$ equation (1.5) turns out to be the only vertex without outgoing arrows except for the isolated Krichever-Novikov equation (1.4). In the scalar setting, we can say that all paths lead to KdV. This is not the case for non-Abelian equations, since the $m \mathrm{KdV}_{2}$ equation also turns out to be an end-point vertex.

\section{DERIVATION OF SUBSTITUTIONS FROM LINEAR PROBLEMS}

The auxiliary linear equations for the non-Abelian KdV equation look same as in the scalar case:

$$
\psi_{x x}=(u-\lambda) \psi, \quad \psi_{t}=u_{x} \psi-(2 u+4 \lambda) \psi_{x} .
$$

However, this can be generalized by replacing the scalar spectral parameter $\lambda$ with a nonAbelian one:

$$
\psi_{x x}=u \psi-\psi \Lambda, \quad \psi_{t}=u_{x} \psi-2 u \psi_{x}-4 \psi_{x} \Lambda
$$

where $\psi, \Lambda \in \mathcal{A}$. Indeed, here $\Lambda$ acts on $\psi$ as the operator of right multiplication $R_{\Lambda}$, and the coefficients $u$ and $u_{x}$ act as operators of left multiplication $L_{u}$ and $L_{u_{x}}$. But, any operators of left and right multiplication commute: $R_{a} L_{b}=L_{b} R_{a}, a, b \in \mathcal{A}$, therefore $R_{\Lambda}$ behaves exactly like the scalar coefficient $\lambda$ when calculating the compatibility condition. It is clear that such a generalization is possible for any zero curvature representation $A_{t}=B_{x}+[B, A]$, where entries of the matrices $A$ and $B$ are elements of $\mathcal{A}$ depending on $\lambda$ and field variables.

We use (3.1) as the starting point for deriving the substitutions from Proposition 2.1.

$P m K d V$ equation. As in the scalar case, the Miura transform is constructed by a particular solution $\psi=y$ corresponding to a fixed value $\Lambda=a \in \mathcal{A}$ :

$$
y_{x x}=u y-y a, \quad y_{t}=u_{x} y-2 u y_{x}-4 y_{x} a .
$$

From the first equation we find $u=y_{x x} y^{-1}+y a y^{-1}$ and the elimination of $u$ from the second equation yields $\operatorname{pmKdV}(a)$. Thus, pmKdV is the equation for the wave function of the Schrödinger operator, and substitution $(2.4)$ is a rewriting of the original linear equation.

Equations $m K d V_{1}$ and $m K d V_{2}$. The next step in the scalar setting is to pass to the logarithmic derivative $f=y_{x} / y$. With non-commutative variables, this can be done in at least two ways, leading to different answers.

The substitution $f=y_{x} y^{-1}$ works only for $a=\alpha \in \mathbb{C}$. In this case, the first equation (3.2) gives the Miura transformation $u=f_{x}+f^{2}+\alpha$ which is the substitution (2.3), and the second equation gives

$$
y_{t}=\left(u_{x}-2 u f-4 \alpha f\right) y=F y, \quad F=f_{x x}+\left[f, f_{x}\right]-2 f^{3}-6 \alpha f .
$$

From equations $y_{x}=f y, y_{t}=F y$ it follows $f_{t}=(D-\operatorname{ad} f)(F)$, which coincides with $\operatorname{mKdV}_{1}(\alpha)$.

If we apply another change $v=y^{-1} y_{x}$, first equation 3.2 becomes $y^{-1} u y=v_{x}+v^{2}+a$. By replacing $u$ in the second equation, we obtain

$$
y_{t}=y\left(y^{-1} u_{x} y-2 y^{-1} u y v-4 v a\right)=y V, \quad V=v_{x x}+2\left[v, v_{x}\right]-2 v^{3}-3 a v-3 v a .
$$

Equations $y_{x}=y v$ and $y_{t}=y V$ imply $v_{t}=(D+\operatorname{ad} v)(V)$, which coincides with $\operatorname{mKdV}_{2}(a)$.

Equation $S K d V$. The next step is to transform equations (3.1) into auxiliary linear problems for $m K d V_{2}$ in such a way that the potential $u$ is replaced by $v$. To do this, it is enough to make the gauge transformation $\psi=y \varphi$ [8]. In other words, we define $\varphi$ as the ratio of solutions for (3.1) and 3.2. A direct calculation leads us to equations

$$
\varphi_{x x}=a \varphi-\varphi \Lambda-2 v \varphi_{x}, \quad \varphi_{t}=4 v(a \varphi-\varphi \Lambda)-2\left(v_{x}+v^{2}+a\right) \varphi_{x}-4 \varphi_{x} \Lambda
$$

where $\varphi, a, \Lambda \in \mathcal{A}$. By construction, the compatibility condition for this pair of equations is equivalent to $\operatorname{mKdV}_{2}(a)$. Now we can do what we did before with the pair (3.1): to write an 
equation for a particular solution $\varphi=z$ corresponding to the value $\Lambda=b$ and for its logarithmic derivative. So, let $z$ satisfy the equations

$$
z_{x x}=a z-z b-2 v z_{x}, \quad z_{t}=4 v(a z-z b)-2\left(v_{x}+v^{2}+a\right) z_{x}-4 z_{x} b .
$$

We find $v$ from the first equation and substitute it into the second one. After simple algebra, this leads to the $\operatorname{SKdV}(a, b, 0)$ equation

$$
z_{t}=z_{x x x}-\frac{3}{2}\left(z_{x x}-a z+z b\right) z_{x}^{-1}\left(z_{x x}+a z-z b\right)-3 a z_{x}-3 z_{x} b
$$

and to the substitution $-2 v=\left(z_{x x}-a z+z b\right) z_{x}^{-1}$ which relates this equation with $\operatorname{mKdV}_{2}(a)$.

In this equation, it is easy to see that the transformation $z \rightarrow z+d$ leads us only to the replacement of the term $a z-z b$ with $a z-z b+c$, where $c=a d-d b$. For arbitrary $a, b, d \in \mathcal{A}$, the element $c$ is arbitrary as well, therefore the equation can be extended to the case of three parameters $\operatorname{SKdV}(a, b, c)$. This leads us to substitution (2.10).

Equations $C D(a, \beta)$ and $C D_{0}(c)$. Since equation $\operatorname{SKdV}(0,0, c)$ contains only derivatives of $z$, it admits the substitution $z_{x}=p$ and we arrive at $\operatorname{CD}_{0}(c)$. For equation $\operatorname{SKdV}(a, b, 0)$, we can use the logarithmic derivative instead, assuming that one of parameters $a$ or $b$ is scalar. In contrast to the pmKdV equation for $y$, this equation is invariant with respect to the transposition and the interchange of parameters, so that both versions $z_{x} z^{-1}$ and $z^{-1} z_{x}$ are on equal footing. For definiteness, let

then equations (3.5) take the form

$$
p=z_{x} z^{-1}, \quad b=\beta \in \mathbb{C},
$$

$$
p_{x}+p^{2}=a-\beta-2 v p, \quad z_{t} z^{-1}=4 v(a-\beta)-2\left(v_{x}+v^{2}+a+2 \beta\right) p .
$$

The first equation is equivalent to the substitution (2.7), and the second equation becomes $z_{t}=P z$ after eliminating $v$, with

$$
P=p_{x x}-\frac{3}{2}\left(p_{x}+p^{2}-a+\beta\right) p^{-1}\left(p_{x}-p^{2}+a-\beta\right)+\left[p, p_{x}\right]-2 p^{3}-6 \beta p .
$$

This yields the equation $p_{t}=(D-\operatorname{ad} p)(P)$, which is $\operatorname{CD}(a-\beta, \beta)$.

\section{BACKLUND TRANSFORMATIONS}

Recall that the scalar tower of modifications $1.5-(1.8)$ can be derived in an alternative way based on Bäcklund transformations [20, 21. For the mKdV equation, the $x$-part of Bäcklund transformations is represented by the dressing chain [25]

$$
f_{n, x}+f_{n+1, x}=f_{n}^{2}-f_{n+1}^{2}+\alpha_{n}-\alpha_{n+1}
$$

generated by compositions of the Miura map and the change $f \rightarrow-f$ which leaves the mKdV equation invariant. Let us introduce a new variable $p_{n}$ and rewrite 4.1 as the system

$$
f_{n}+f_{n+1}=p_{n}, \quad f_{n}-f_{n+1}=\frac{p_{n, x}-\alpha_{n}+\alpha_{n+1}}{p_{n}},
$$

then both $f_{n}$ and $f_{n+1}$ are easily expressed through $p_{n}$ and $p_{n, x}$. This is the substitution $(1.7) \rightarrow 1.6$, up to the values of parameters, and moreover, we obtain the chain for $p_{n}$

$$
\left(p_{n} p_{n+1}\right)_{x}=p_{n} p_{n+1}\left(p_{n}-p_{n+1}\right)+\left(\alpha_{n}-\alpha_{n+1}\right) p_{n+1}+\left(\alpha_{n+1}-\alpha_{n+2}\right) p_{n} .
$$

The CD equation for $p_{n}$ is obtained by applying the found substitutions to the result of differentiating the relation $p_{n}=f_{n}+f_{n+1}$ in virtue of the $\operatorname{mKdV}$ equations for $f_{n}$ and $f_{n+1}$. Moreover, the invariance with respect to the shift $n \rightarrow n+1$ guarantees that $p_{n+1}$ also satisfies the CD equation, so that the chain (4.2) defines the $x$-part of Bäcklund transformations for this equation.

This trick can be repeated once more by introducing the variable $w_{n}=p_{n} p_{n+1}$ and this leads us to the substitution $(1.8) \rightarrow(1.7)$. Further modification is not possible, since the chain equation for $w_{n}$ does not have some functional structure required for this, see [21]. 
What about this procedure in the non-Abelian setting? One can easily see that chain 4.1 still works, since the form of the Miura map $(2.3)$ is the same as for the scalar counterparts. Therefore, (4.1) defines the Bäcklund transformations for the non-Abelian $m \operatorname{KdV}_{1}(\alpha)$ equation. However, introducing the variable $p_{n}=f_{n}+f_{n+1}$ now gives nothing for the simple reason that the difference of non-Abelian squares $f_{n}^{2}-f_{n+1}^{2}$ is not factorizable. Unfortunately, this nice scheme fails already at the first step.

Nevertheless, the chain of Bäcklund transformations for the non-Abelian CD equation can still be constructed, but for this we have to use the substitution (2.7) from CD to $m K d V_{2}$ and the change $p \rightarrow-p^{t}$ which leaves CD equation invariant. Hence it follows that there are two different substitutions between the $\mathrm{CD}(a-\beta, \beta)$ and the $\mathrm{mKdV}_{2}(a)$ equations:

$$
-2 v=p^{-1}\left(-p_{x}+p^{2}-a+\beta\right) \quad \text { and } \quad-2 v=\left(p_{x}+p^{2}-a+\beta\right) p^{-1} .
$$

In these substitutions, $\beta$ can be changed, but $a$ is fixed, since this parameter is contained in the target $\mathrm{mKdV}_{2}(a)$ equation. This gives rise to a sequence of substitutions

$$
-2 v_{n}=p_{n}^{-1}\left(-p_{n, x}+p_{n}^{2}-a+\beta_{n}\right)=\left(p_{n+1, x}+p_{n+1}^{2}-a+\beta_{n+1}\right) p_{n+1}^{-1},
$$

from which we obtain the chain

$$
\left(p_{n} p_{n+1}\right)_{x}=p_{n}\left(p_{n}-p_{n+1}\right) p_{n+1}-\left(a-\beta_{n}\right) p_{n+1}+p_{n}\left(a-\beta_{n+1}\right) .
$$

By construction, it defines the $x$-part of Bäcklund transformations between equations $\mathrm{CD}\left(a-\beta_{n}, \beta_{n}\right)$. Of course, this remains true also for scalar variables, but note that the scalar version of (4.3) differs from 4.2): in one equation the signs of two linear terms coincide, and in the other they are opposite. This distinction is due to different methods which we used to construct the chains, and it turns out that one method admits a non-Abelian generalization, while the other does not.

In conclusion, we remark that Miura maps (2.3) and (2.7) admit non-local generalizations. Relations like (3.3), which were obtained under assupmtion $a=\alpha \in \mathbb{C}$, can be obtained also for $a \in \mathcal{A}$, at the expense of introducing an additional variable. Let $f=y_{x} y^{-1}, g=y a y^{-1}$ then relations 3.2 imply

$$
u=f_{x}+f^{2}+g, \quad y_{t}=F y, \quad F=f_{x x}+\left[f, f_{x}\right]-2 f^{3}-3 f g-3 g f,
$$

and we arrive to the following system for $f$ and $g$ :

$$
f_{t}=(D-\operatorname{ad} f)(F)=f_{x x x}-3\left(f^{2}+g\right) f_{x}-3 f_{x}\left(f^{2}+g\right), \quad g_{t}=[F, g], \quad g_{x}=[f, g] .
$$

The variable $g$ can be viewed as a nonlocality defined by the latter equation which plays the role of a constraint. The invariance of all relations with respect to the change $(u, f, g) \rightarrow\left(u^{t},-f^{t}, g^{t}\right)$ implies that there is also another Miura map $u=-f_{x}+f^{2}+g$. The composition of these two substitutions gives rise to the dressing chain

$$
f_{n, x}+f_{n+1, x}=f_{n}^{2}-f_{n+1}^{2}+g_{n}-g_{n-1}, \quad g_{n, x}=\left[f_{n}, g_{n}\right],
$$

which defines the general Darboux transformations for the non-Abelian Schrödinger operator [6] and the $x$-part of Bäcklund transformations for the above system for $f$ and $g$.

In a similar way, relations $(3.6)$ obtained under assumption $b=\beta \in \mathbb{C}$ can be generalized for the case $b \in \mathcal{A}$, by introducing the additional variable $h=z b z^{-1}$. This leads to the system

$$
\begin{gathered}
p_{t}=(D-\operatorname{ad} p)(P), \quad h_{t}=[P, h], \quad h_{x}=[p, h], \\
P=p_{x x}-\frac{3}{2}\left(p_{x}+p^{2}-a+h\right) p^{-1}\left(p_{x}-p^{2}+a-h\right)+\left[p, p_{x}\right]-2 p^{3}-3 p h-3 h p
\end{gathered}
$$

and to the chain

$$
\left(p_{n} p_{n+1}\right)_{x}=p_{n}\left(p_{n}-p_{n+1}\right) p_{n+1}-\left(a-h_{n}\right) p_{n+1}+p_{n}\left(a-h_{n+1}\right), \quad h_{n, x}=\left[p_{n}, h_{n}\right],
$$

which defines the $x$-part of Bäcklund transformations for this system and the Darboux transformations for spectral problem (3.4). 


\section{REFERENCES}

1. M. Wadati, T. Kamijo. On the extension of inverse scattering method // Prog. Theor. Phys. 52, 397-414 (1974).

2. F. Calogero, A. Degasperis. Nonlinear evolution equations solvable by the inverse spectral transform. II // Il Nuovo Cim. 39B:1, 1-54 (1977).

3. L. Martinez Alonso, E. Olmedilla. Trace identities in the inverse scattering transform method associated with matrix Schrödinger operators // J. Math. Phys. 23:11, 2116-2121 (1982).

4. V.A. Marchenko. Nonlinear equations and operator algebras. Reidel, Boston (1988).

5. V.M. Goncharenko, A.P. Veselov. Monodromy of the matrix Schrödinger equations and Darboux transformations // J. Phys. A 31:23, 5315-5326 (1998).

6. A.A. Suzko. Intertwining technique for the matrix Schrödinger equation// Phys. Lett. A 335:2-3, 88-102 (2005).

7. F.A. Khalilov, E.Ya. Khruslov. Matrix generalization of the modified Korteweg-de Vries equation // Inverse Problems 6:2, 193-204 (1990).

8. Q.P. Liu, C. Athorne. Comment on 'Matrix generalization of the modified Korteweg-de Vries equation'// Inverse Problems 7:5, 783-785 (1991).

9. B.A. Kupershmidt. KP or mKP. Noncommutative mathematics of Lagrangian, Hamiltonian, and integrable systems. AMS, Providence, RI (2000).

10. V.E. Adler, V.V. Sokolov. Non-Abelian evolution systems with conservation laws // Math. Phys. Anal. Geom. 24, 7 (2021).

11. S.I. Svinolupov, V.V. Sokolov. Evolution equations with nontrivial conservation laws // Funct. Anal. Appl. 16:4, 317-319 (1982).

12. A.V. Mikhailov, A.B. Shabat, V.V. Sokolov. The symmetry approach to classification of integrable equations // in "What is integrability?", ed. V.E. Zakharov, Springer-Verlag, 115-184 (1991).

13. S.I. Svinolupov, V.V. Sokolov. Deformations of Jordan triple systems and integrable equations // Theor. Math. Phys. 108:3, 1160-1163 (1996).

14. V.E. Adler, V.V. Sokolov. On matrix Painlevé II equations // Theor. Math. Phys. 207:2, 560-571 (2021).

15. S. Carillo, M.L. Schiavo, E. Porten, C. Schiebold. A novel noncommutative KdV-type equation, its recursion operator, and solitons // J. Math. Phys. 59:4, 043501 (2018).

16. S.I. Svinolupov, V.V. Sokolov, R.I. Yamilov. On Bäcklund transformations for integrable evolution equations // Sov. Math. Dokl. 28, 165-168 (1983).

17. I.M. Krichever, S.P. Novikov. Holomorphic bundles over algebraic curves and nonlinear equations // Russ. Math. Surveys 35:6, 53-79 (1980).

18. F. Calogero, A. Degasperis. Reduction technique for matrix nonlinear evolution equations solvable by the spectral transform // J. Math. Phys. 22:1, 23-31 (1981).

19. A.S. Fokas. A symmetry approach to exactly solvable evolution equations // J. Math. Phys. 21:6, 1318-1325 (1980).

20. R.I. Yamilov. Invertible changes of variables generated by Bäcklund transformations // Theor. Math. Phys. 85:3, 1269-1275 (1990).

21. R.I. Yamilov. On the construction of Miura type transformations by others of this kind // Phys. Lett. A 173:1, 53-57 (1993).

22. A.B. Borisov, S.A. Zykov. The dressing chain of discrete symmetries and proliferation of nonlinear equations // Theor. Math. Phys. 115:2, 530-541 (1998).

23. S.Ya. Startsev. Differential substitutions of the Miura transformation type // Theor. Math. Phys. 116:3, 1001-1010 (1998).

24. S.I. Svinolupov. Jordan algebras and integrable systems // Funct. Anal. Appl. 27:4, 257-265 (1993).

25. A.P. Veselov, A.B. Shabat. Dressing chains and the spectral theory of the Schrödinger operators // Funct. Anal. Appl. 27:2, 81-96 (1993).

Vsevolod Eduardovich Adler,

L.D. Landau Institute for Theoretical Physics,

Akademika Semenova av., 1A,

142432, Chernogolovka, Moscow Region, Russia

E-mail: adler@itp.ac.ru 